\title{
AN ARITHMETICAL CHARACTERIZATION OF DECOMPOSITION METHODS IN BANACH SPACES VIA SUPPLEMENTED SUBSPACES
}

\author{
ELÓI MEDINA GALEGO \\ Department of Mathematics - IME, University of São Paulo, São Paulo 05315-970, Brazil \\ e-mail:eloi@ime.usp.br
}

(Received 8 December, 2004; accepted 5 May, 2005)

\begin{abstract}
Let $X$ and $Y$ be Banach spaces such that each one is isomorphic to a complemented subspace of the other. In 1996, W. T. Gowers solved the SchroederBernstein Problem for Banach spaces by showing that $X$ is not necessarily isomorphic to $Y$. In this paper, we give suitable conditions on $X, Y$, the supplemented subspaces of $Y$ in $X$ and of $X$ in $Y$ to yield that $X$ is isomorphic to $Y$. In other words, we obtain generalizations of Pełczyński's decomposition method via supplemented subspaces. In order to determine all the possible generalizations, we introduce the notion of Mixed Schroeder-Bernstein Quadruples for Banach spaces. Then, we use some Banach spaces constructed by W. T. Gowers and B. Maurey in 1997 to characterize them.
\end{abstract}

2000 Mathematics Subject Classification. 46B03, 46B20.

1. Introduction. Let $X$ and $Y$ be Banach spaces. We write $X \sim Y$ if $X$ is isomorphic to $Y$ and $X \nsim Y$ otherwise. If $n \in \mathbb{N}^{*}=\{1,2,3, \cdots\}$, then $X^{n}$ denotes the sum of $n$ copies of $X, X \oplus X \oplus \cdots \oplus X$. It will be useful to denote $X^{0}=\{0\}$. We recall that $Y$ is isomorphic to a complemented subspace of $X$ if there exists a Banach space $A$ such that $X \sim Y \oplus A$. In this case, we say that $A$ is a supplemented subspace of $X$ associated to $Y$.

Suppose now that $X$ and $Y$ are Banach spaces such that there exist Banach spaces $A$ and $B$ satisfying

$$
\left\{\begin{array}{l}
X \sim Y \oplus A \\
Y \sim X \oplus B .
\end{array}\right.
$$

In 1996, W. T. Gowers [11] solved the so-called Schroeder-Bernstein Problem for Banach spaces by showing that $X$ is not necessarily isomorphic to $Y$; see also [2], [3], [4], [5], [7] and [12]. However Pełczyński’s decomposition method [1, page 64] which has played an important role in the isomorphic theory of classical Banach spaces, states that $X \sim Y$ if we add to (1) the conditions $X \sim X^{2}$ and $Y \sim Y^{2}$. The starting point of this paper is the fact that these conditions can be rewritten in terms of the supplemented subspaces $A$ and $B$ associated to $Y$ and $X$ respectively as follows:

$$
\left\{\begin{array}{l}
X \sim Y^{2} \oplus A^{2} \\
Y \sim X^{2} \oplus B^{2} .
\end{array}\right.
$$

So if we are interested in investigating the influence of the supplemented subspaces in decomposition methods similar to Pełczyński's decomposition method, it is natural 
to ask whether it is possible to obtain all pairs of conditions similar to that of (2) which added to (1) also yield $X \sim Y$. To be more precise, in the spirit of [6], we make the following definition. See Remark 2.2 below.

Definition 1.1. A quadruple $(p, q, r, s)$ in $\mathbb{N}^{*}$ is a Mixed Schroeder-Bernstein Quadruple for Banach spaces (in short, MSBQ) if $X \sim Y$ for any Banach spaces $X, Y$, $A$ and $B$ satisfying (1) and the conditions

$$
\left\{\begin{array}{l}
X \sim Y^{p} \oplus A^{q}, \\
Y \sim X^{r} \oplus B^{s} .
\end{array}\right.
$$

Thus Pełczyński's decomposition method states that $(2,2,2,2)$ is a MSBQ. On the other hand, the above mentioned result of $\mathrm{W}$. T. Gowers shows that $(1,1,1,1)$ is not a MSBQ.

The aim of this work is to show that thanks to the development of the isomorphic theory of Banach spaces in the last years, it is possible to characterize the quadruples in $\mathbb{N}^{*}$ which are MSBQ; see Theorem 3.1. Indeed, we shall see that a certain family of Banach spaces constructed by W. T. Gowers and B. Maurey in 1997 (see Remark 2.1) is large enough to provide such characterization (see Lemmas 3.2 and 3.4).

2. Preliminaries. We start by recalling some results on Banach spaces which are isomorphic to complemented subspaces of each other. If $m, n$ are integers, then $m \mid n$ means that $m$ divides $n$ and $\operatorname{gcd}(m, n)$ denotes their greatest common divisor.

REMARK 2.1. In [12, p. 563] Banach spaces $X_{t}$, for every $t \in \mathbb{N}, t \geq 2$, were constructed having the following property: $X_{t}^{m} \sim X_{t}^{n}$, with $m, n \in \mathbb{N}^{*}$, if and only if $m$ is congruent to $n$ modulo $t$.

REMARK 2.2. Following [6, Definition 1.1] a quadruple $(p, q, r, s)$ in $\mathbb{N}$, with $p+$ $q \geq 2$ and $r+s \geq 2$ is said to be a Schroeder-Bernstein Quadruple for Banach spaces (in short, SBQ) if $X \sim Y$ for every pair of Banach spaces $X$ and $Y$ isomorphic to complemented subspaces of each other and satisfying the following Decomposition Scheme

$$
\left\{\begin{array}{l}
X \sim X^{p} \oplus Y^{q} \\
Y \sim X^{r} \oplus Y^{s}
\end{array}\right.
$$

$\Delta=(p-1)(s-1)-r q$ is called the discriminant of the quadruple $(p, q, r, s)$. The main theorem of [6] states that a quadruple $(p, q, r, s)$ in $\mathbb{N}$ with $p+q \geq 2$ and $r+s \geq 2$ is a SBQ if and only if $\Delta \neq 0, \Delta \mid(p+q-1)$ and $\Delta \mid(r+s-1)$.

REMARK 2.3. In [8] a quadruple $(p, q, r, s)$ in $\mathbb{N}$, with $p+q \geq 2$ and $r+s \geq 2$ was said to be a Nearly Schroeder-Bernstein Quadruple for Banach spaces (in short, NSBQ) if $X^{m} \sim Y^{n}$ for some $m, n \in \mathbb{N}^{*}$ whenever the Banach spaces $X$ and $Y$ are isomorphic to complemented subspaces of each other and satisfy the Decomposition Scheme (4). It was shown in [8, Theorem 3.2] and [9, Theorem 2.3] that a quadruple $(p, q, r, s)$ in $\mathbb{N}$, with $p+q \geq 2$ and $r+s \geq 2$ is a NSBQ if and only if its discriminant $\Delta$ is different from zero.

REMARK 2.4. Let $X$ and $Y$ be Banach spaces isomorphic to complemented subspaces of each other. In [7, Remark 3.5] it was proved that if $X^{p} \sim X^{p-1} \oplus Y$ for some $p \in \mathbb{N}, p \geq 2$ and $X \sim X^{r} \oplus Y^{s}$ for some $r, s \in \mathbb{N}, r+s \geq 2$, then $X \sim Y$. 
REMARK 2.5. [5, Lemma 2.2] Suppose that $X$ and $Y$ are Banach spaces isomorphic to complemented subspaces of each other with

$$
X^{i} \oplus Y^{j} \sim X^{k} \oplus Y^{l},
$$

for some $i, j, k, l \in \mathbb{N}$. If $k<i$ and $j<l$, then $X^{m} \sim Y^{n}$ for some $m, n \in \mathbb{N}^{*}$.

REMARK 2.6. Fix two Banach spaces $X$ and $Y$ from the class of spaces constructed in [10], as was done in [5]. By [5] there exist Banach spaces $E$ and $Z$ such that

(a) $E=Z \oplus X, Z=E \oplus Y$ and $Z \sim Z^{2}$ [5, p. 31].

(b) $Z \nsim Z \oplus X^{m}$ for every $m \in \mathbb{N}^{*}[\mathbf{5}$, Theorem 3.4].

\section{Characterization of the Mixed Schroeder-Bernstein Quadruples for Banach} spaces. First of all it is clear from the symmetry of Definition 1.1 that $(r, s, p, q)$ is a MSBQ if and only if $(p, q, r, s)$ is one. So Theorem 3.1 provides a characterization of the quadruple in $\mathbb{N}^{*}$ which are MSBQ. The proof of this result is quite long and it will be done by proving several propositions.

TheOREM 3.1. Let $(p, q, r, s)$ be a quadruple in $\mathbb{N}^{*}$.

(a) Suppose that $p<q$.

If $p=1$ and $q=2$, then $(p, q, r, s)$ is a MSBQ for every $r, s \in \mathbb{N}^{*}$.

If $p=1$ and $q>2$ then $(p, q, r, s)$ is a MSBQ if and only if $r=1$ and $\operatorname{gcd}(q-1$, $s-1)=1$.

If $p>1$ then $(p, q, r, s)$ is a MSBQ if and only if either $(r, s)=(1,2)$ or $q=p+1, r \geq 2$, $(r-1) \mid(p-1)$ and $s=1$.

(b) Suppose that $p \geq q$ and $r \geq s$ and put $D=(q-1)(s-1)-(r-s)(p-q)$. Then $(p, q, r, s)$ is a MSBQ if and only if $p \geq 2, r \geq 2, D \neq 0, D \mid(p-1)$ and $D \mid(r-1)$.

The following four lemmas play an important role in the proof of our theorem. They are closely related to the Banach spaces $X_{t}$ mentioned in Remark 2.1.

Lemma 3.2. Let $p, q, r, s \in \mathbb{N}^{*}$ and suppose that there exist $i, j, u \in \mathbb{N}$ with $u \geq 2$, $0 \leq i<j<u$ satisfying

(a) $u \mid(i(1-p)+j(p-q))$;

(b) $u \mid(i(1-r)+j(s-1))$.

Then $(p, q, r, s)$ is not a MSBQ.

Proof. Since $(j-i)+(u-j)-(u-i)=0$ and $(u-i)+j-(j-i)=u$, we have by the property of $X_{u}$ mentioned in Remark 2.1 that

$$
\left\{\begin{array}{l}
X_{u}^{u-i} \sim X_{u}^{j-i} \oplus X_{u}^{u-j} \\
X_{u}^{j-i} \sim X_{u}^{u-i} \oplus X_{u}^{j}
\end{array}\right.
$$

Now note that the condition (a) implies that $u$ divides $p(j-i)+q(u-j)-(u-i)$. Furthermore, it follows from condition (b) that $u$ also divides $r(u-i)+s j-(j-i)$. Thus again by Remark 2.1 we deduce

$$
\left\{\begin{array}{l}
X_{u}^{u-i} \sim X_{u}^{p(j-i)} \oplus X_{u}^{q(u-j)} \\
X_{u}^{j-i} \sim X_{u}^{r(u-i)} \oplus X_{u}^{s j} .
\end{array}\right.
$$


But $X_{u}^{u-i}$ is not isomorphic to $X_{u}^{j-i}$, because $u$ does not divide $(u-i)-(j-i)=$ $u-j$. Consequently $(p, q, r, s)$ is not a MSBQ.

By interchanging $(p, q)$ with $(r, s)$ in Lemma 3.2, we obtain the following lemma which will be later referred to.

LemmA 3.3. Let $p, q, r, s \in \mathbb{N}^{*}$ and suppose that there exist $i, j, u \in \mathbb{N}$ with $u \geq 2$, $0 \leq i<j<u$ satisfying

(a) $u \mid(i(1-r)+j(r-s))$;

(b) $u \mid(i(1-p)+j(q-1))$.

Then $(p, q, r, s)$ is not a MSBQ.

Lemma 3.4. Let $p, q, r, s \in \mathbb{N}^{*}$ with $(q-p)(r-1)+(p-1)(s-1) \geq 2$ and suppose that there exist integers $\alpha$ and $\beta$ satisfying

(a) $\beta(p-q) \geq \alpha(s-1)$;

(b) $\beta(1-q)<\alpha(s-r)$;

(c) $\alpha(1-r)+\beta(p-1)<(q-p)(r-1)+(p-1)(s-1)$.

Then $(p, q, r, s)$ is not a MSBQ.

Proof. Put $u=(q-p)(r-1)+(p-1)(s-1) \geq 2$ and consider the linear system

$$
\left\{\begin{array}{l}
i(1-p)+j(p-q)=\alpha u \\
i(1-r)+j(s-1)=\beta u .
\end{array}\right.
$$

The only solution of (5) is $i=\beta(p-q)-\alpha(s-1)$ and $j=\alpha(1-r)+\beta(p-1)$. It follows from (a), (b) and (c) that $0 \leq i<j<u$. Moreover, clearly $u \mid(i(1-p)+j(p-q))$ and $u \mid(i(1-r)+j(s-1))$. Therefore Lemma 3.2 implies that $(p, q, r, s)$ is not a MSBQ.

By interchanging $(p, q)$ with $(r, s)$ in Lemma 3.4 and noting that $(s-r)(p-1)+$ $(r-1)(q-1)=(q-p)(r-1)+(p-1)(s-1)$ we get:

Lemma 3.5. Let $p, q, r, s \in \mathbb{N}^{*}$ with $(q-p)(r-1)+(p-1)(s-1) \geq 2$ and suppose that there exist integers $\alpha$ and $\beta$ satisfying

(a) $\beta(r-s) \geq \alpha(q-1)$;

(b) $\beta(1-s)<\alpha(q-p)$;

(c) $\alpha(1-p)+\beta(r-1)<(q-p)(r-1)+(p-1)(s-1)$.

Then $(p, q, r, s)$ is not a MSBQ.

Our main task is now to show how to use these lemmas to prove Theorem 3.1. Theorem 3.1(a) is an immediate consequence of Propositions 3.6, 3.7, 3.8, 3.10, 3.11, 3.12, 3.13 and 3.14. Theorem 3.1(b) follows directly from Propositions 3.15 and 3.16.

PROPOSITION 3.6. If $p=1$ and $q=2$, then $(p, q, r, s)$ is a MSBQ for every $r, s \in \mathbb{N}^{*}$.

Proof. Let $X, Y, A$ and $B$ be Banach spaces satisfying (1) and (3). Thus

$$
X \sim Y \oplus A^{2} \sim(Y \oplus A) \oplus A \sim X \oplus A .
$$

Consequently

$$
X \sim Y \oplus A \sim X \oplus A \oplus B \sim X \oplus B \sim Y .
$$

Hence $(p, q, r, s)$ is a MSBQ. 
Proposition 3.7. If $p=1, q>2$ and $r>1$, then $(p, q, r, s)$ is not a MSBQ for any $s \in \mathbb{N}^{*}$.

Proof. We distinguish four cases: $r=s ; s=1 ; s>1$ and $r>s ; s>1$ and $r<s$.

Case 1. $r=s$. Take $i=0, j=1$ and $u=q-1 \geq 2$ and observe that $u \mid(i(1-r)+$ $j(r-s))=0$ and $u \mid(i(1-p)+j(q-1))=q-1$. So Lemma 3.3 implies that $(p, q, r, s)$ is not a MSBQ.

Case 2. $s=1$. Take $i=0, j=1$ and $u=q-1 \geq 2$ in and note that $u \mid(i(1-$ $p)+j(p-q))=1-q$ and $u \mid(i(1-r)+j(s-1))=0$. Thus Lemma 3.2 implies that $(p, q, r, s)$ is not a MSBQ.

Case 3. $s>1$ and $r>s$. Take $i=s-1, j=r-1$ and $u=(r-1)(q-1) \geq$ 2. Observe that $u \mid(i(1-p)+j(p-q))=(r-1)(1-q))$ by the definition of $u$ and $u \mid(i(1-r)+j(s-1))=0$ by the choices of $i$ and $j$. According to Lemma $3.2(p, q, r, s)$ is not a MSBQ.

Case 4. $s>1$ and $r<s$. There are two subcases: $r=2$ and $r \geq 3$.

Subcase 4.1. $r=2$. Since $\{s-1\} \cup\{s-2-k: 0 \leq k<q-2\}$ is a set with $q-1$ consecutive numbers, it follows that either $(q-1) \mid(s-1)$ or $(q-1) \mid(s-2-k)$ for some $0 \leq k<q-2$.

Subcase 4.1.1. $(q-1) \mid(s-1)$. Take $i=0, j=1$ and $u=q-1$ and observe that $u \mid(i(1-p)+j(p-q))=(1-q)$ and $u \mid(i(1-r)+j(s-1))=(s-1)$. So Lemma 3.2 implies that $(p, q, r, s)$ is not a MSBQ.

Subcase 4.1.2. $(q-1) \mid(s-2-k)$ for some $0 \leq k<q-2$. Take $i=k, j=q-2$ and $u=q-1$ and note that $u \mid(i(1-r)+j(r-s))=-i+(q-2)(2-s)=s-2-k+$ $(q-1)(2-s)$ and $u \mid(i(1-p)+j(q-1))=(q-2)(q-1)$. Thus Lemma 3.3 implies that $(p, q, r, s)$ is not a MSBQ.

Subcase 4.2. $r \geq 3$. Thus the hypothesis $(q-p)(r-1)+(p-1)(s-1) \geq 2$ of Lemma 3.4 is verified. Take $u=(q-1)(r-1)$. Since $q \geq 3$ and $r \geq 3$, it follows that $(q-1) /(q-2) \leq 2 \leq r-1$. So

$$
q-1 \leq(q-2)(r-1)=(q-2)(s-1)-(q-2)(s-r) .
$$

Let $m$ be the greatest number in $\mathbb{N}$ satisfying

$$
m(q-1) \leq(q-2)(s-r)
$$

Therefore

$$
(q-2)(s-r)<(m+1)(q-1) .
$$

According to (6) and (7), we have

$$
(m+1)(q-1)=m(q-1)+(q-1) \leq(q-2)(s-1) .
$$

Let $\alpha=2-q$ and $\beta=m+1$. By (8) and (9), it follows that (a) and (b) of Lemma 3.4 are verified. Moreover, it is easy to check that $\alpha(1-r)+\beta(p-1)<$ $(q-p)(r-1)+(p-1)(s-1)$. Consequently $(p, q, r, s)$ is not a MSBQ. 
PROPOSITION 3.8. If $p=1, q>2$ and $r=1$, then $(p, q, r, s)$, with $s \in \mathbb{N}^{*}$, is a MSBQ if and only if $\operatorname{gcd}(q-1, s-1)=1$.

Proof. Suppose first that $u=\operatorname{gcd}(q-1, s-1) \geq 2$ and take $i=0$ and $j=1$. So $u \mid(i(1-r)+j(r-s))=1-s$ and $u \mid(k(1-p)+j(q-1))=q-1$. Thus Lemma 3.3 implies that $(p, q, r, s)$ is not a MSBQ.

Conversely, assume now that $\operatorname{gcd}(q-1, s-1)=1$ and $X, Y, A$ and $B$ are Banach spaces satisfying (1) and (3).

First note that if $X \sim X \oplus E$ for some Banach space $E$, then adding $E$ to both sides of this isomorphism we obtain

$$
X \sim X \oplus E \sim X \oplus E^{2} .
$$

Hence by induction we deduce that for every $t \in \mathbb{N}^{*}$,

$$
X \sim X \oplus E^{t} .
$$

Now by the well-known Bezout's theorem there exist $m, n \in \mathbb{N}$ such that $m(q-1)=$ $1+n(s-1)$ or $n(s-1)=1+m(q-1)$.

Case 1. $m(q-1)=1+n(s-1)$. By (1) and (3) we have

$$
X \sim(Y \oplus A) \oplus A^{q-1} \sim X \oplus A^{q-1} .
$$

Therefore by (10) with $E=A^{q-1}$ and $t=m$, we obtain

$$
X \sim X \oplus A^{m(q-1)} .
$$

On the other hand, by (1) we know that $X \sim X \oplus A \oplus B$. So again by (10), with $E=A \oplus B$ and $t=n(s-1)$, we conclude that

$$
X \sim X \oplus A^{n(s-1)} \oplus B^{n(s-1)} .
$$

Moreover, by (3) we infer that

$$
X \sim Y \oplus A \sim X \oplus B^{s} \oplus A \sim(X \oplus A \oplus B) \oplus B^{s-1} \sim X \oplus B^{s-1} .
$$

Hence once more by (10) with $E=B^{s-1}$ and $t=n$, we have

$$
X \sim X \oplus B^{n(s-1)} .
$$

According to (11), (12) and (13),

$X \sim X \oplus A^{m(q-1)} \oplus B^{n(s-1)} \sim X \oplus A^{1+n(s-1)} \oplus B^{n(s-1)} \sim X \oplus A^{n(s-1)} \oplus B^{n(s-1)} \oplus A$ $\sim X \oplus A$.

Thus by (1), we see that

$$
X \sim Y \oplus A \sim X \oplus A \oplus B \sim X \oplus B \sim Y .
$$

Case 2. $n(s-1)=1+m(q-1)$. According to (11) and (13), we have

$X \sim X \oplus A^{m(q-1)} \oplus B^{n(s-1)} \sim X \oplus A^{m(q-1)} \oplus B^{1+m(q-1)} \sim X \oplus A^{m(q-1)} \oplus B^{m(q-1)} \oplus B$ $\sim X \oplus B \sim Y$.

Therefore $(p, q, r, s)$ is a MSBQ.

In the sequel we shall need the following lemma. 
Lemma 3.9. For every $q, s \in \mathbb{N}$, with $q \geq 3$ and $s \geq 3$, there exists $m \in \mathbb{N}^{*}$ such that

$$
\left(\frac{s-2}{s-1}\right)(q-2) \leq m \leq\left(\frac{s-2}{s-1}\right)(q-1) .
$$

Proof. There exist $j \in \mathbb{N}^{*}$ and $k \in \mathbb{N}, 0 \leq k \leq s-2$ such that $q-2=j(s-1)-k$. It will be convenient to distinguish the cases $k=0$ and $k \neq 0$.

Case 1. $k=0$. Therefore

$$
\left(\frac{s-2}{s-1}\right)(q-2)=j(s-2)<j(s-2)+\left(\frac{s-2}{s-1}\right)=\left(\frac{s-2}{s-1}\right)(q-1) .
$$

Thus it is enough to take $m=j(s-2)$.

Case 2. $k \neq 0$. Since $1 \leq k<s-1$, we have

$$
\begin{aligned}
\left(\frac{s-2}{s-1}\right)(q-2) & =j(s-2)-k\left(\frac{s-2}{s-1}\right)<j(s-2)-(k-1) \\
& \leq j(s-2)-(k-1)\left(\frac{s-2}{s-1}\right)=\left(\frac{s-2}{s-1}\right)(q-1) .
\end{aligned}
$$

Hence it suffices to take $m=j(s-2)-(k-1)$.

Proposition 3.10. If $1<p<q$ and $s \neq 1$, then $(p, q, r, s)$, with $r \in \mathbb{N}^{*}$, is a MSBQ if and only if $(r, s)=(1,2)$.

Proof. Suppose first that $(r, s) \neq(1,2)$. There are several cases to consider: $s=2$ and $r=2 ; s=2$ and $r>2 ; s \geq 3$ and $r=1 ; s \geq 3$ and $r \geq 2$. Note that in every one of these cases the hypothesis $(q-p)(r-1)+(p-1)(s-1) \geq 2$ of Lemmas 3.4 and 3.5 is verified.

Case 1. $s=2$ and $r=2$. Take $\alpha=1$ and $\beta=0$. Since $q>2$, we have that (a), (b) and (c) of Lemma 3.5 are verified. So $(p, q, r, s)$ is not a MSBQ.

Case 2. $s=2$ and $r>2$. Take $\alpha=p-q$ and $\beta=0$. Having in mind that $p>1$, apply Lemma 3.5 to conclude that $(p, q, r, s)$ is not a MSBQ.

Case 3. $s \geq 3$ and $r=1$. There are two subcases: $p=2$ and $p \geq 3$.

Subcase 3.1. $p=2$. Let $m \in \mathbb{N}^{*}$ as in the Lemma 3.9. If

$$
\left(\frac{s-2}{s-1}\right)(q-2) \leq m<\left(\frac{s-2}{s-1}\right)(q-1)
$$

then take $\alpha=-m$ and $\beta=s-2$. Therefore (a), (b) and (c) of Lemma 3.4 are verified. Thus $(p, q, r, s)$ is not a MSBQ.

Otherwise,

$$
\left(\frac{s-2}{s-1}\right)(q-2)<m \leq\left(\frac{s-2}{s-1}\right)(q-1) .
$$


Thus take $\alpha=m$ and $\beta=2-s$. Hence (a), (b) and (c) of Lemma 3.5 are verified. Consequently $(p, q, r, s)$ is not a MSBQ.

Subcase 3.2. $p \geq 3$. Since $s \geq 3$, we deduce that

$$
\left(\frac{s-2}{s-1}\right)(q-1)-\left(\frac{s-2}{s-1}\right)(q-p)=\left(\frac{s-2}{s-1}\right)(p-1) \geq 1 .
$$

Let $m$ be the greatest number in $\mathbb{N}$ such that

$$
m \leq\left(\frac{s-2}{s-1}\right)(q-p)
$$

Therefore by (14) and (15), we conclude that

$$
\left(\frac{s-2}{s-1}\right)(q-p)<m+1 \leq\left(\frac{s-2}{s-1}\right)(q-1) .
$$

Let $\alpha=m+1$ and $\beta=2-s$. By (16) we see that (a), (b) and (c) of Lemma 3.5 are verified. Hence $(p, q, r, s)$ is not a MSBQ.

Case 4. $s \geq 3$ and $r \geq 2$. Take $\alpha=p-q$ and $\beta=s-2$. So $\beta(p-q) \geq \alpha(s-1)$.

Moreover, since $p>1$,

$$
\left(\frac{s-r}{s-2}\right) \leq 1<\left(\frac{q-1}{q-p}\right)
$$

Hence $(s-2)(q-1)>(q-p)(s-r)$, that is, $\beta(1-q)<\alpha(s-r)$.

Now observe that $\alpha(1-r)+\beta(p-1)=(p-q)(1-r)+(s-2)(p-1)<$ $(q-p)(r-1)+(p-1)(s-1)$. Thus Lemma 3.4 implies that $(p, q, r, s)$ is a MSBQ.

Finally, assume that $(r, s)=(1,2)$. Proceeding as in the proof of Proposition 3.6 we see that $(p, q, r, s)$ is a MSBQ.

PROPOSITION 3.11. If $1<p<q, s=1$ and $q \neq p+1$, then $(p, q, r, s)$ is not $a$ MSBQ for every $r \in \mathbb{N}^{*}$.

Proof. We will distinguish two cases: $r=1$ and $r \geq 2$.

Case 1. $r=1$. Take $i=0, j=1$ and $u=q-p \geq 2$. So $u \mid(i(1-p)+j(p-q))=$ $p-q$ and $u \mid(i(1-r)+j(s-1))=0$. According to Lemma 3.2 $(p, q, r, s)$ is not a MSBQ.

Case 2. $r \geq 2$. Since $(q-p)(r-1) \geq 2$, it suffices to put $\alpha=p-q+1, \beta=0$ and to apply Lemma 3.4 to see that $(p, q, r, s)$ is not a MSBQ. MSBQ.

Proposition 3.12. If $p>1, s=1, q=p+1$ and $r=1$, then $(p, q, r, s)$ is not a

Proof. Since $q-1 \geq 2$, it is enough to take $i=0, j=1, u=q-1$ and to apply Lemma 3.3 to conclude that $(p, q, r, s)$ is not a MSBQ, because $u \mid(i(1-r)+j(r-s))=$ 0 and $u \mid(i(1-p)+j(q-1))=q-1$. 
Proposition 3.13. If $p>1, s=1, q=p+1, r>2$ and $r-1$ does not divide $p-1$, then $(p, q, r, s)$ is not a MSBQ.

Proof. First note that the hypothesis $(q-p)(r-1)+(p-1)(s-1) \geq 2$ of Lemma 3.5 is verified. Next, there are two cases: $p<r$ and $p>r$.

Case 1. $p<r$. Take $\alpha=\beta=1$. Since $r-1 \geq p$ and $(r-1)+(1-p)<(r-1)$, it follows that (a), (b) and (c) of Lemma 3.5 are verified. So $(p, q, r, s)$ is not a MSBQ.

Case 2. $p>r$. Let $\alpha$ be the first number in $\mathbb{N}$ such that $\alpha(r-1) \geq(p-1)$. So $\alpha \geq 2$ and $(\alpha-1)(r-1)<(p-1)$. Since $r-1$ does not divide $p-1$, it follows that $\alpha(r-1) \neq(p-1)$. Therefore $\alpha(r-1) \geq p$. Take $\beta=1$. Thus (a), (b) and (c) of Lemma 3.5 are verified. Hence $(p, q, r, s)$ is not a MSBQ.

Proposition 3.14. If $p>1, s=1, q=p+1, r \geq 2$ and $r-1$ divides $p-1$, then $(p, q, r, s)$ is a MSBQ.

Proof. First note that (1) and (3) imply

$$
Y \sim X^{r} \oplus B \sim X^{r-1} \oplus X \oplus B \sim X^{r-1} \oplus Y .
$$

Therefore, by (1), we deduce

$$
X \sim Y \oplus A \sim X^{r-1} \oplus Y \oplus A \sim X^{r-1} \oplus X \sim X^{r} .
$$

Next, let $m \in \mathbb{N}^{*}$ such that $p-1=m(r-1)$. Again by (1) and (3) we get

$$
X \sim Y^{p} \oplus A^{p} \oplus A \sim X^{p} \oplus A \sim X^{p-1} \oplus X \oplus A \sim X^{m(r-1)} \oplus X \oplus A .
$$

Now adding $Y$ on the both sides of (19) we obtain by (17)

$$
X \oplus Y \sim X^{r-1} \oplus X^{r-1} \oplus \cdots \oplus X^{r-1} \oplus Y \oplus X \oplus A \sim Y \oplus X \oplus A \sim X^{2} .
$$

It follows from (18), (20) and Remark 2.1 that $X \sim Y$. So $(p, q, r, s)$ is a MSBQ.

Proposition 3.15. If $p=q=1$ and $r \geq s$, then $(p, q, r, s)$ is not a MSBQ.

Proof. We will distinguish two cases: $r<2$ and $r \geq 2$.

Case 1. $s=1$. Take $i=0, j=1$ and $u=2$. By Lemma 3.2 ( $p, q, r, s)$ is not a MSBQ.

Case 2. $s=2$. There are three subcases: $r=2 ; r=3$ and $r \geq 4$.

Subcase 2.1. $r=2$. Take $i=0, j=1, u=2$. Thus $u \mid(i(1-r)+j(r-s))=0$ and $u \mid(i(1-p)+j(q-1))=0$. By Lemma 3.3 we conclude that $(p, q, r, s)$ is not a MSBQ.

Subcase 2.2. $r=3$. Take $i=1, j=2, u=3$. Then, $u \mid(i(1-r)+j(r-s))=0$ and $u \mid(i(1-p)+j(q-1))=0$. According to Lemma 3.3 $(p, q, r, s)$ is not a MSBQ.

Subcase 2.3. $r \geq 4$. Therefore $r-s \geq 2$. Let $i=0, j=1$ and $u=r-s$. So $u \mid(i(1-r)+j(r-s))=r-s$ and $u \mid(i(1-p)+j(q-1))=0$. Thus Lemma 3.3 implies that $(p, q, r, s)$ is not a MSBQ. 
Case 3. $s \geq 3$. Take $i=0, j=1$ and $u=s-1$. Hence $u \mid(i(1-p)+j(p-q))=0$ and $u \mid(i(1-r)+j(s-1))=s-1$. Now it is enough to apply Lemma 3.2 to deduce that $(p, q, r, s)$ is not a MSBQ.

Proposition 3.16. Let $p, q, r, s \in \mathbb{N}^{*}$ with $p \geq 2, r \geq 2, p \geq q$ and $r \geq s$. Put $D=$ $(q-1)(s-1)-(r-s)(p-q)$. Then $(p, q, r, s)$ is a MSBQ if and only if $D \neq 0, D \mid(p-1)$ and $D \mid(r-1)$.

Proof. Notice first that if $X, Y, A$ and $B$ are Banach spaces satisfying (1) and (3), then

$$
\begin{gathered}
X \sim Y^{p-q} \oplus Y^{q} \oplus A^{q} \sim Y^{p-q} \oplus(Y \oplus A)^{q} \sim X^{q} \oplus Y^{p-q} \\
Y \sim X^{r-s} \oplus X^{s} \oplus B^{s} \sim X^{r-s} \oplus(X \oplus B) \sim X^{r-s} \oplus Y^{s} .
\end{gathered}
$$

Conversely, if $X, Y, A$ and $B$ are Banach spaces satisfying (1), (21) and (23), then clearly these spaces verify (3).

Therefore, since $p \geq 2$ and $r \geq 2$, it follows that $(p, q, r, s)$ is a MSBQ if and only if $(q, p-q, r-s, s)$ is a Schroeder-Bernstein Quadruple. Since the discriminant $\Delta$ of $(q, p-q, r-s, s)$ is equal to $D$, it suffices to use the characterization of SchroederBernstein Quadruple mentioned in Remark 2.2 to complete the proof.

The proof of Theorem 3.1 is now complete.

4. Characterization of the Nearly Mixed Schroeder-Bernstein Quadruples for Banach spaces. Inspired by Theorem 3.1 and Remark 2.3 we introduce the following definition:

Definition 4.1. A quadruple $(p, q, r, s)$ in $\mathbb{N}^{*}$ is a Nearly Mixed SchroederBernstein Quadruple for Banach spaces (in short, NMSBQ) if for every Banach spaces $X, Y, A$ and $B$ satisfying the conditions (1) and (3), we have that $X^{m} \sim Y^{n}$, for some $m, n \in \mathbb{N}^{*}$.

We end the paper by providing the characterization of the quadruples in $\mathbb{N}^{*}$ which are NMSBQ.

THEOREM 4.2. Let $(p, q, r, s)$ be a quadruple in $\mathbb{N}^{*}$.

(a) Suppose that $p<q$ or $r<s$. Then $(p, q, r, s)$ is a NMSBQ.

(b) Suppose that $p \geq q$ and $r \geq s$ and put $D=(q-1)(s-1)-(r-s)(p-q)$. Then $(p, q, r, s)$ is $a$ MSBQ if and only if $p \geq 2, r \geq 2$ and $D \neq 0$.

Proof. (a) By symmetry it is enough to consider the case $p<q$. Let $X, Y, A$ and $B$ be Banach spaces satisfying (1) and (3). Thus adding $Y^{q}$ to both sides of the first isomorphism of (3) and using (1) we have

$$
X \oplus Y^{q} \sim Y^{p} \oplus A^{q} \oplus Y^{q} \sim Y^{p} \oplus X^{q} .
$$

Since $p<q$, it follows that $q>1$. Hence Remark 2.5 implies that $X^{m} \sim Y^{n}$ for some $m, n \in \mathbb{N}^{*}$. So $(p, q, r, s)$ is NMSBQ.

(b) We distinguish the cases $p=1$ and $r=1 ; p=1$ and $r=2 ; p=2$ and $r=1$; $p \geq 2$ and $q \geq 2$. Again by symmetry it suffices to consider the cases 1,2 and 4 . 
Case 1. $p=1$ and $r=1$. Thus $q=1$ and $s=1$. Let $E, Z, X$ and $Y$ be the Banach spaces mentioned in Remark 2.6. It follows from Remark 2.6(a) that

$$
\left\{\begin{array}{l}
Z \sim E \oplus Y \\
E \sim Z \oplus X .
\end{array}\right.
$$

But according to Remark 2.6(b), we obtain for every $m, n \in \mathbb{N}^{*}$,

$$
E^{m} \sim Z^{m} \oplus X^{m} \sim Z \oplus X^{m} \nsim Z \sim Z^{n} .
$$

Consequently $(1,1,1,1)$ is not a NMSBQ.

Case 2. $p=1$ and $r=2$. There are two subcases: $s=1$ and $s=2$.

Subcase 2.1. $s=1$. Again Remark 2.6(a) implies that

$$
\left\{\begin{array}{l}
Z \sim E \oplus Y \\
E \sim Z^{2} \oplus X .
\end{array}\right.
$$

Hence by $(23)$ we deduce that $(1,1,2,1)$ is not a NMSBQ.

Subcase 2.2. $s=2$. Once more by Remark 2.6(a) we see that

$$
\left\{\begin{array}{l}
E \sim Z \oplus X \\
Z \sim E^{2} \oplus Y^{2} .
\end{array}\right.
$$

Thus by (23) we conclude that $(1,1,2,2)$ is not a NMSBQ.

Case 4. $p \geq 2, r \geq 2$. Let $X, Y, A$ and $B$ be Banach spaces satisfying (1). By (21) and (22) we know that (3) is equivalent to

$$
\left\{\begin{array}{l}
X \sim X^{q} \oplus Y^{p-q} \\
Y \sim X^{r-s} \oplus Y^{s} .
\end{array}\right.
$$

Therefore $(p, q, r, s)$ is a NMSBQ if and only if $(q, p-q, r-s, s)$ is a NSBQ. Then, the result follows from Remark 2.3.

AcKnOwLEDGEMENT. The author would like to thank the referee for useful comments and suggestions.

\section{REFERENCES}

1. P. G. Casazza, The Schroeder-Bernstein property of Banach spaces, Contemporary Mathematics 85 (1989), 61-77.

2. E. M. Galego, How to generate new Banach spaces non-isomorphic to their cartesian squares, Bull. Polish Acad. Sci. Math. 47 (1999), 21-25.

3. E. M. Galego, Banach spaces complemented in each other without isomorphic finite sums, Bull. Polish Acad. Sci. Math. 50 (2002), 1-9.

4. E. M. Galego, On solutions to the Schroeder-Bernstein problem for Banach spaces, Arch. Math. (Basel) 74 (2002), 299-307.

5. E. M. Galego, The Schroeder-Bernstein index for Banach spaces, Studia Math. 164 (2004), 29-38.

6. E. M. Galego, An arithmetic characterization of decomposition methods in Banach spaces similar to Pełczyński's decomposition method, Bull. Polish Acad. Sci. Math. 52 (2004), 273-282. 
7. E. M. Galego, On pairs of Banach spaces which are isomorphic to complemented subspaces of each other, Colloq. Math. 101 (2004), 279-287.

8. E. M. Galego, On extensions of Pełczyński’s decomposition method in Banach spaces, Arch. Math. (Basel), to appear.

9. E. M. Galego, Characterization of the Nearly Schroeder-Bernstein Quadruples for Banach spaces. Preprint.

10. W. T. Gowers, The unconditional basic sequence problem, J. Amer. Math. Soc. 6(1993), 851-874.

11. W. T. Gowers, A solution to the Schroeder-Bernstein problem for Banach spaces, Bull. London Math. Soc. 28 (1996), 297-304.

12. W. T. Gowers and B. Maurey, Banach spaces with small spaces of operators, Math. Ann. 307 (1997), 543-568. 